ANL/AOD/CP-101379

\title{
Initial Nonintercepting Measurements of Subpicosecond Bunches Using Coherent Diffraction Radiation
}

\author{
A. H. Lumpkin, N. S. Sereno, G. A. Decker, D. W. Rule*
}

\author{
Argonne National Laboratory \\ 9700 South Cass Avenue \\ Argonne, IL 60439 \\ *Carderock Division \\ Naval Surface Warfare Center \\ West Bethesda, MD 20817
}

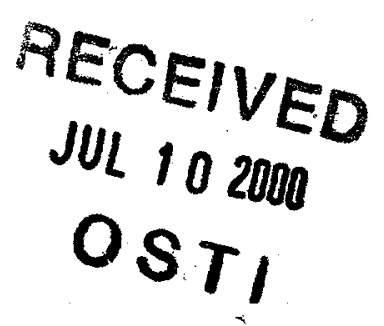

Presented at the

$9^{\text {th }}$ Beam Instrumentation Workshop

Cambridge, MA

May 8-11, 2000

The submitted manuscript has been created by the University of Chicago as Operator of Argonne National Laboratory ("Argonne") under Contract No. W-31-109-ENG-38 with the U.S. Department of Energy. The U.S. Government retains for itself. and others acting on its behalf, a paid-up. nonexclusive. irrevocable worldwide license in said anticle to reproduce. prepare derivative works, distribute copies to the public, and perform publicly and display publicly, by or on behalf of the Government. 


\section{DISCLAIMER}

This report was prepared as an account of work sponsored by an agency of the United States Government. Neither the United States Government nor any agency thereof, nor any of their employees, make any warranty, express or implied, or assumes any legal liability or responsibility for the accuracy, completeness, or usefulness of any information, apparatus, product, or process disclosed, or represents that its use would not infringe privately owned rights. Reference herein to any specific commercial product, process, or service by trade name, trademark, manufacturer, or otherwise does not necessarily constitute or imply its endorsement, recommendation, or favoring by the United States Government or any agency thereof. The views and opinions of authors expressed herein do not necessarily state or reflect those of the United States Government or any agency thereof. 


\section{DISCLAIMER}

Portions of this document may be illegible in electronic image products. Images are produced from the best available original document. 


\title{
Initial Nonintercepting Measurements of Subpicosecond Bunches Using Coherent Diffraction Radiation
}

\author{
A. H. Lumpkin, N. S. Sereno, G. A. Decker and D. W. Rule ${ }^{+}$ \\ Advanced Photon Source, Argonne National Laboratory, \\ 9700 South Cass Avenue, Argonne, IL 60439 \\ + Carderock Division, Naval Surface Warfare Center \\ West Bethesda, MD 20817
}

\begin{abstract}
We report initial measurements of the subpicosecond (sub-ps) bunch structure in a $40-\mathrm{MeV}$ linac beam using the autocorrelation of far infrared (FIR) coherent diffraction radiation (CDR). Since the radiation was generated as the charged particles passed through a slit or aperture in a metal screen oriented at 45 degrees to the beam direction, this is a nonintercepting technique. A compact Michelson interferometer was used to obtain the autocorrelation of the backward CDR with a Golay cell used as the FIR-mm wave detector. The longitudinal shape was calculated from the autocorrelation using the minimal phase approximation. A model was developed and used to calculate the CDR spectral emissions. The experimental results, analysis, and model are presented. Complementary measurements were also made with coherent transition radiation (CTR).
\end{abstract}

\section{INTRODUCTION}

The interest in high-brightness electron beams for accelerator-based research continues to stimulate the development of diagnostics of short bunches [1]. As noted in various workshops [2-5], the need for nonintercepting techniques has been invoked to address high average current devices or to allow on-line measurements for operating experiments. In this work we present the first (to our knowledge) measurements that are nonintercepting and identify sub-ps beam structure using the autocorrelation of FIR coherent diffraction radiation. In earlier work by Shibata et al., the forward CDR and electron beam were intercepted by the downstream radiation pickoff mirror [6]. In this case we used the backward CDR generated as the $40-\mathrm{MeV}$ linac beam macropulse passed through a slit or aperture in a metal screen oriented at 45 degrees to the beam direction. A compact Michelson interferometer [7] was used to obtain the autocorrelation, and the longitudinal shape was calculated using the minimal phase approximation described by Lai and Sievers [8]. A model was also developed to calculate the expected CDR spectral emissions from the back of two 
metal strips separated by a $5-\mathrm{mm}$ vertical gap centered on the beam axis. We also performed complementary measurements using coherent transition radiation and a zero-phasing rf technique.

\section{EXPERIMENTAL BACKGROUND}

The Advanced Photon Source (APS) injector linear accelerator (linac) consists of an S-band thermionic if gun and $\alpha$ magnet that allow a macropulse of 8 to $40 \mathrm{~ns}$ in length to be injected into the accelerator as shown in Fig. 1 [9]. The micropulses each have about 20 to $50 \mathrm{pC}$ of charge so bunch lengths of less than $500 \mathrm{fs}$ (FWHM) are needed in order to reach the target 100 -A peak currents. In gun optimization studies [10] we have found specific regimes where the core of the beam has transverse emittances of the order of 5 to $10 \pi \mathrm{mm}$ mrad. The nominal beam properties at the station where these measurements were performed are given in Table 1.

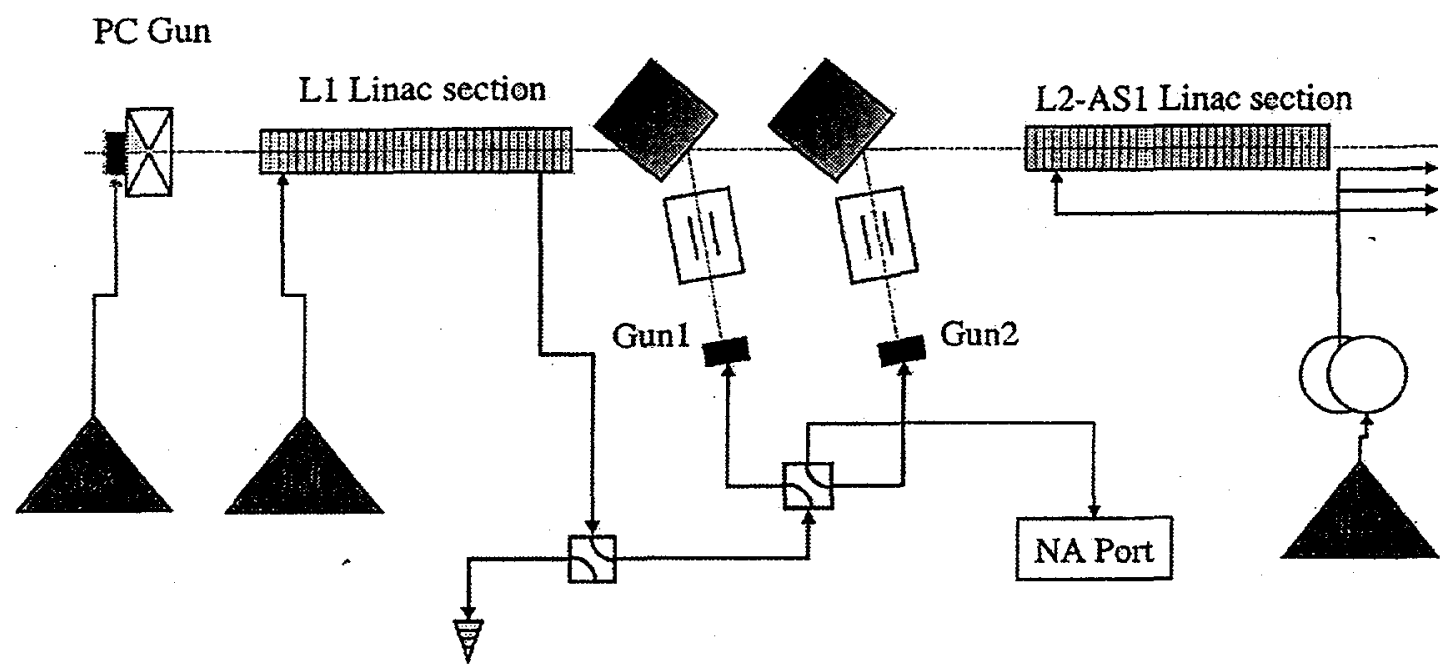

FIGURE 1. A schematic of the injection linac for the APS. In these experiments the beam from the thermionic if gun \#2 was accelerated to $40 \mathrm{MeV}$ by the L2AS1 accelerating structure after which the diagnostics station was located. (Courtesy of J. Lewellen, ANL)

Table 1: APS Linac Beam Properties at Station-1 in the Low-Emittance Mode (If Thermionic Gun)

\begin{tabular}{|l|l|}
\hline rf frequency $(\mathrm{MHz})$ & 2856 \\
\hline Beam energy $(\mathrm{MeV})$ & $40-50$ \\
\hline Micropulse charge $(\mathrm{pC})$ & $20-50$ \\
\hline Micropulse duration $(\mathrm{ps})$ & $0.2-1.0$ (FWHM) \\
\hline Macropulse length $(\mathrm{ns})$ & 40 \\
\hline Macropulse repetition Rate $(\mathrm{Hz})$ & 6 \\
\hline normalized emittance $(\pi \mathrm{mm}$ mrad) & $\sim 5-10(1 \sigma)$ \\
\hline
\end{tabular}


The standard beam profile station on the linac uses an intercepting Chromox screen with a video camera. For investigating bright beams we have modified the station at $40 \mathrm{MeV}$. In this case one actuator has a Ce-doped YAG single crystal with mirror at $\mathbf{4 5}$ degrees to the beam for beam position and profile information with high conversion efficiency. The second actuator with a kinematic positioner has a metal screen with a 5-mm-tall slit aperture machined into it beginning $1.5 \mathrm{~mm}$ above the beam centerline when it is inserted. This design was a compromise dictated by the need to use an alignment laser to position properly the Michelson interferometer and detector below the beamline. The alignment laser was injected into the beamline upstream of the location so that the angle of specular reflection could be determined from the metal screen. By steering the e-beam on the centerline of the beamline, the solid metal is intercepted for OTR or CTR studies. Steering the beam vertically a few $\mathrm{mm}$ allowed the beam to pass through the aperture and to be viewed on the downstream electron spectrometer. With this steering the CDR experiments were performed. In addition to the visible beam image downstream, we also used the If beam position monitor (BPM) sum signal as a relative current monitor before and after the CTR/CDR screen. We tracked these signals during the course of the interferometer mirror position scans.

The CTR and CDR are generated from the metal surface or "metal strips," respectively, seen by the beam. The radiation leaves the beam pipe through a crystalline quartz window and is colliminated by a $100-\mathrm{mm}$ focal length crystalline quartz (z-cut) plano-convex lens. A Michelson interferometer is used to analyze the spectrum of the coherent radiation or, equivalently, to perform an autocorrelation of the emitted transition radiation pulse as described in Reference 7. An Inconel-coated beamsplitter is used to generate the two beams used in the autocorrelation. One beam's path length can be adjusted by a computer-controlled mirror stage. A Golay detector, Model OAD-7, obtained from QMC Instruments Limited was used as the broadband FIR detector. Signal levels of a few hundred $\mathrm{mV}$ were obtained with 200-mA macropulse average current. The analog data were processed and digitized with an APS-built gated integrator module. EPICS was the platform used to track the signal versus mirror position, $\alpha$-magnet current, or scraper position.

\section{ANALYTICAL BACKGROUND}

A model was developed and used to calculate the CDR spectral emissions from the back of two metal strips separated by a vertical gap centered on the beam axis, as schematically shown in Fig. 2. The spectral emissions were calculated for nominal cases at $50 \mathrm{MeV}, 100-\mathrm{pC} / \mu$ pulse, 80 pulses. The calculation was benchmarked on measured spectra reported by Shibata et al. [6]. Sensitivities of the CDR spectrum to beam offset from the center of the slot and to slots of various widths were assessed. Model guidance supported the 5-mm vertical gap for our nominal 1-ps case at 40 to 50 $\mathrm{MeV}$. A brief outline of the approach is given below. 


\section{Coherent Diffraction Radiation}

Coherent radiation by a bunch of electrons can be expressed as the product of a term representing the radiation process for a single particle and a term, which takes into account how much of the total charge in the bunch radiates together, constructively in phase. Thus the spectral, angular distribution of the radiation can be expressed as

$$
\frac{d^{2} W}{d \omega d \Omega}=\frac{d^{2} W_{1}}{d \omega d \Omega} B_{N}
$$

where $d^{2} W_{1} / d \omega d \Omega$ is the spectral angular distribution for the single particle radiation process, either transition radiation (TR), synchrotron radiation, or diffraction radiation (DR), for example. In the present case $d^{2} W_{1} / d \omega d \Omega$ is taken to be the single-particle diffraction radiation process. $B_{\mathrm{N}}$ is the coherence factor for a bunch of $\mathrm{N}$ electrons. The term $B_{\mathrm{N}}$ is related to the square of the Fourier transform of the spatial distribution of the bunch:

$$
\mathrm{B}_{\mathrm{N}}=\mathrm{N}+\mathrm{N}(\mathrm{N}-1) \mathrm{F}(k) \text {, }
$$

with

$$
\mathrm{F}(k)=\left[\mathrm{Q}^{-1} \mathfrak{I}\{\rho(r)\}\right]^{2} .
$$

Here

$$
\mathfrak{S}\{\rho(r)\}=\int \rho(r) e^{-\mathrm{iK} \cdot \mathrm{R}} \mathrm{d} r
$$

is the bunch charge form factor, i.e., the Fourier transform of the charge distribution of total charge $\mathrm{Q}=\mathrm{Ne}$. The first term in Eq. (2) yields the incoherent radiation produced by $\mathrm{N}$ electrons in the bunch, while the second term gives the coherent production, which is proportional to $\mathrm{N}^{2}$.

\section{Bunch Form Factor}

A simple Gaussian model for the bunch form factor is used to calculate CDR for various cases relevant to the APS linac. The Gaussian charge density is taken to be

$$
\rho(r)=\rho(\mathrm{x}, \mathrm{y}, \mathrm{z})=\frac{1}{\sqrt{2 \pi \sigma_{\mathrm{x}}^{2}}} \mathrm{e}^{-\mathrm{x}^{2} / 2 \sigma_{\mathrm{x}}^{2}} \frac{1}{\sqrt{2 \pi \sigma_{\mathrm{y}}^{2}}} \mathrm{e}^{-\mathrm{y}^{2} / 2 \sigma_{\mathrm{y}}^{2}} \frac{1}{\sqrt{2 \pi \sigma_{\mathrm{z}}^{2}}} \mathrm{e}^{-\mathrm{z}^{2} / 2 \sigma_{\mathrm{z}}^{2}},
$$


where $\sigma_{x}, \sigma_{y}$, and $\sigma_{z}$ are the rms bunch parameters for the transverse beam dimensions $(x, y)$ and the longitudinal dimension $z$, i.e., along the beam axis. The value $\sigma_{z}$ is to be determined by observing the CDR.

The Fourier transform of $\rho(r)$ is just

$$
\mathfrak{S}\{\rho(r)\}=Q \mathrm{e}^{-\left(\sigma_{\mathrm{x}} \mathrm{k}_{\mathrm{x}}\right)^{2} / 2} \mathrm{e}^{-\left(\sigma_{\mathrm{y}} \mathrm{k}_{\mathrm{y}}\right)^{2} / 2} \mathrm{e}^{-\left(\sigma_{z} \mathrm{k}_{\mathrm{z}}\right)^{2} / 2}
$$

Using this in Eq. (3), $F(k)$ can be expressed as

$$
F(k)=e^{-\left(\sigma_{x} k_{x}\right)^{2}} e^{-\left(\sigma_{y} k_{y}\right)^{2}} e^{-\left(\sigma_{z} k_{z}\right)^{2}}
$$

Considering the transverse, i.e., $x$ - and $y$-terms above, if the beam is radially symmetric, then $\sigma^{2}=\sigma_{x}^{2}+\sigma_{y}^{2}=2 \sigma_{x}^{2}$. The component vectors above are defined as:

$$
\begin{gathered}
\mathrm{k}_{\mathrm{x}}=\mathrm{k} \sin (\theta) \cos (\varphi)=\mathrm{k} \sin \left(\theta_{\mathrm{x}}\right) \approx \mathrm{k} \theta_{\mathrm{x}} \\
\mathrm{k}_{\mathrm{y}}=\mathrm{k} \sin (\theta) \sin (\varphi)=\mathrm{k} \sin \left(\theta_{\mathrm{y}}\right) \approx \mathrm{k} \theta_{\mathrm{y}} \\
\mathrm{k}_{\mathrm{z}}=\mathrm{k} \cos (\theta) \approx \mathrm{k}
\end{gathered}
$$

where the last term on the right of the equations is valid for the relativistic beams that we shall consider in this study because the angles of interest are of the order of $1 / \gamma$, i.e., the inverse of the Lorentz factor. For a radially symmetric beam, we can write the transverse exponents in Eq. (6) above as

$$
\sigma_{x}^{2} k_{x}^{2}+\sigma_{y}^{2} k_{y}^{2}=\frac{\sigma^{2}}{2} k^{2} \theta^{2}
$$

where $\theta^{2} \approx \theta_{x}^{2}+\theta_{y}^{2}$. Note that for $F(k)$ to be sizeable, the beam rms radius $\sigma$ and the wavelength of interest $\lambda$ must be such that for angles $\theta$ of order $1 / \gamma$,

$$
\sigma \leq \sqrt{2} \gamma \lambda
$$

where $\lambda=\lambda / 2 \pi$. The wavelengths of interest are determined by the longitudinal part of $F(k)$ such that the rms bunch length

$$
\sigma_{\mathrm{z}} \leq \lambda
$$

Comparing Eqs. (9) and (10), it can be seen that there is a relativistic effect that allows the beam radial dimensions to be of the order of $\gamma$ times $\lambda / 2 \pi$ and radiate coherently, while the wavelength for coherent radiation must be greater than the longitudinal 
dimension of the bunch $\left(\sim 2 \sigma_{z}\right)$. Since the number of electrons $N$ in a bunch is typically quite large, $F(k)$ can actually be much less than unity and there will still be coherent radiation.

A computer program to calculate coherent diffraction radiation as a function of wavelength was written and applied to the general cases of interest here [11]. In Fig. 3 the calculated spectra of CDR from a slit for a $50-\mathrm{MeV}$ electron beam comprising $8 \mathrm{nC}$ in a macropulse for bunch lengths from $\sigma_{t}=0.2$ to $6 \mathrm{ps}$ are shown. As expected, the form factor results in significant enhancements of radiation at wavelengths about three times longer than the bunch length. For the nominal 1-ps case, the effects of beam offset up to $1.0 \mathrm{~mm}$ from the center of the aperture are shown as minimal in Fig. 4. The clear aperture width is considered in Fig. 5. The 2.5-mm and 5-mm cases give somewhat more signal than the large apertures. We used the $5-\mathrm{mm}$ vertical clear aperture in these experiments.

\section{EXPERIMENTAL RESULTS AND ANALYSIS PROCEDURES}

Most of the data discussed in this section were obtained in November 1999. The gun rf gradient was higher than the setting used for injection into the storage ring. The higher gradient with the proper $\alpha$ magnet and low energy scraper setting can be used to select a core of the beam. In this case the beam was first evaluated using the CTR signals from Station-1 steering on the centerline.

The autocorrelation was obtained, and a fast Fourier transform was performed to assess the spectral content. Using the observed amplitudes from 20 to $40 \mathrm{~cm}^{-1}$ as reference, the amplitudes for longer wavelengths were extrapolated. The corrected spectrum was used in recalculating the autocorrelation [12]. The longitudinal profile was calculated from the autocorrelation using the minimal phase approximation of Lai and Sievers [8]. The CTR data are shown in Fig. 6a. The bunch distribution shows a spike in the leading edge (supported by a zero-phasing rf technique) with a FWHM distribution of about $450 \mathrm{fs}$. We then steered the beam upward and sent the beam through the aperture. The CDR FIR signal level was lower but still sufficient to obtain an autocorrelation. The CDR data are displayed in Fig. 6b. A similar longitudinal distribution was obtained using the same analysis. It is noted that the single-particle DR production is explicitly wavelength dependent. This difference between TR and DR is not yet included in the analysis of the data. The differences become even smaller at higher gamma. The energy distribution was observed downstream of the CDR aperture's location, and the rf BPM sum signals from locations before and after the aperture were used to confirm the transmission of beam. In addition, we have evaluated a $4 \mathrm{~mm} \times 11 \mathrm{~mm}$ aperture as a CDR generator at $217 \mathrm{MeV}$ and the potential use of waveguide and microwave techniques for transport and analysis. This latter work is in the preliminary stage. 


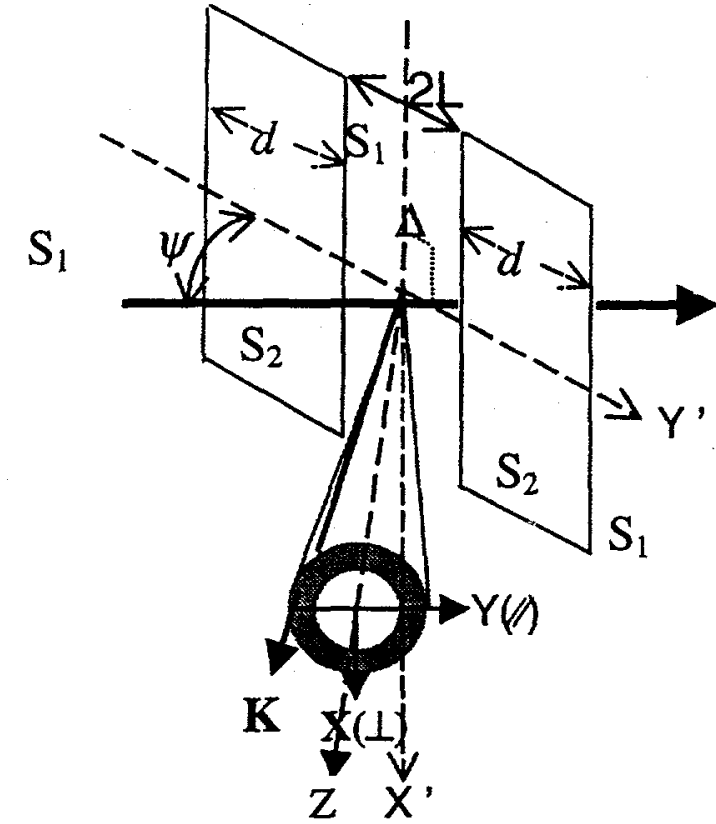

FIGURE 2. Schematic of geometry for diffraction radiation production by two finite width, infinitely long, reflecting strips. Here, the $y^{\prime}$ axis is in the plane containing the velocity vector $v$ and the normal to the plane of the aperture. Radiation is emitted in the direction of $k$.

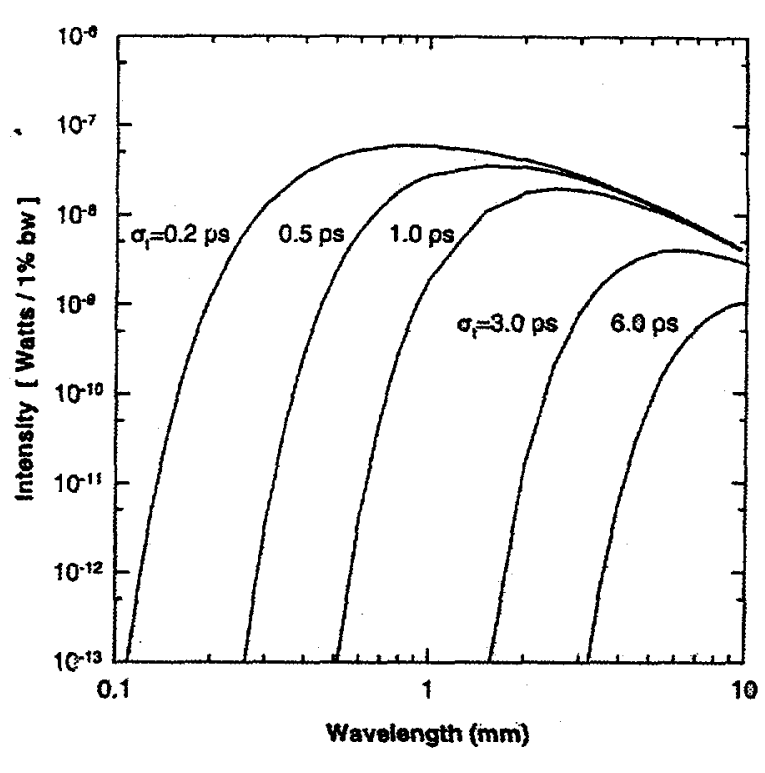

FIGURE 3. Calculated spectrum of CDR from a slit for a $50-\mathrm{MeV}$ electron beam with $8 \mathrm{nC}$ total charge and bunch lengths of $\sigma_{t}=0.2,0.5,1.0$, 3.0 , and 6.0 ps.

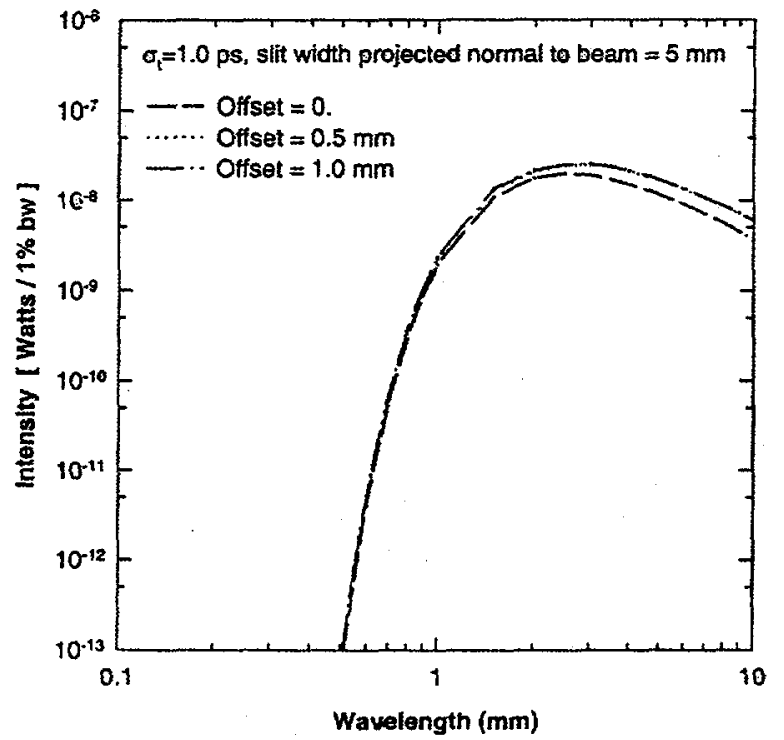

FIGURE 4. Calculated spectrum of CDR due to beam offsets from the center of the slit.

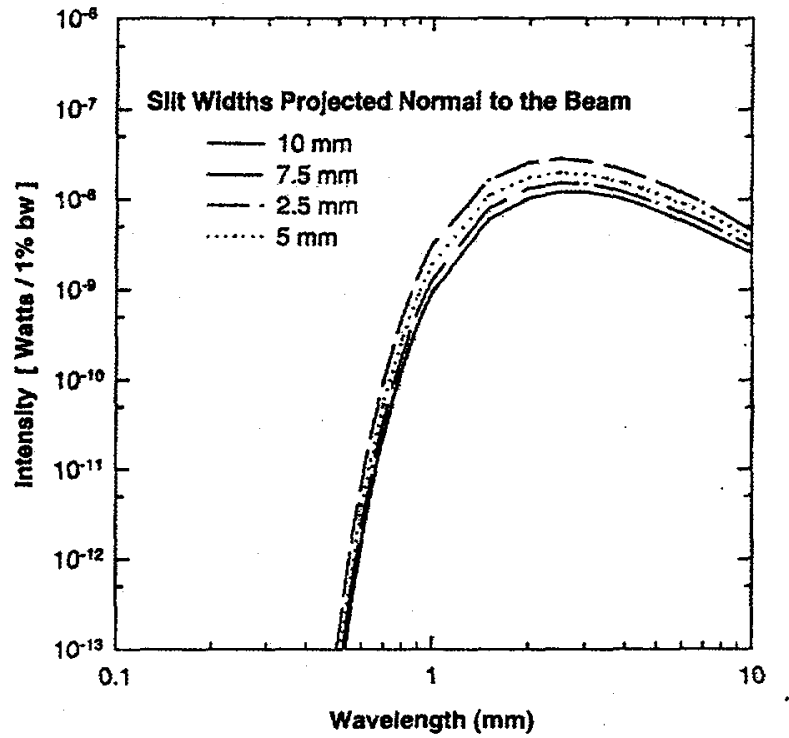

FIGURE 5. Calculated spectrum of CDR produced by slits of various widths for a 1 -ps bunch length at $50 \mathrm{MeV}$. 

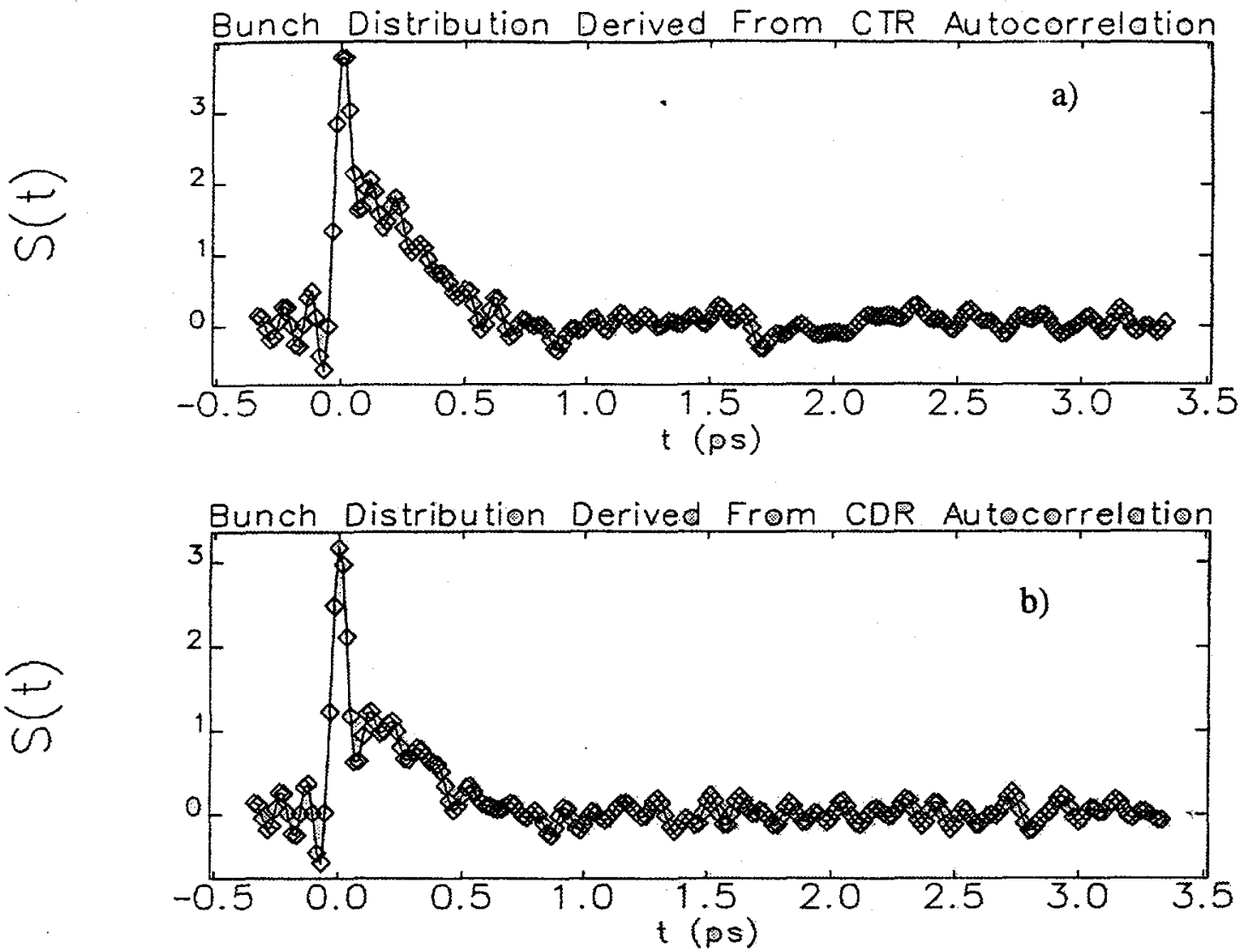

FIGURE 6. Calculated longitudinal bunch distribution from the experimental autocorrelation using the minimal phase technique: a) using CTR and b) using CDR. The bunch distribution is sub-0.5 ps (FWHM) for the 40-MeV beam.

\section{SUMMARY}

In summary, we have obtained the first, to our knowledge, nonintercepting measurements of sub-ps electron-beam bunch lengths using backward CDR. The measurements were done with $\gamma<100$ and for very small charges in a micropulse (20$50 \mathrm{pC}$ ). The total charge in the 115 micropulses in the macropulse was 3-4 $\mathrm{nC}$. We have subsequently obtained a CDR interferogram with only 25 micropulses in the macropulse and about $1 \mathrm{nC}$ total charge. Further work is planned at different charge levels and beam energies, but it is anticipated that the technique is applicable to a number of experiments currently relying on the intercepting CTR-based technique. The scaling indicates that at higher $\gamma$ the CDR approaches the CTR signal strengths for an appropriate aperture so even the Linac Coherent Light Source project may benefit. 


\section{ACKNOWLEDGMENTS}

This work is supported by the U.S. Department of Energy, Office of Basic Sciences, under Contract No. W-31-109-ENG-38. The authors acknowledge W. Berg for engineering assistance, N. Arnold for software support, and J. Galayda for project support.

\section{REFERENCES}

1. Micro Bunches, Eds. E. B. Blum, M. Dienes, J. B. Murphy, AIP Conf. Proc. 367, Woodbury, N.Y. (1996).

2. Alex H. Lumpkin, "On the Path to the Next Generation of Light Sources," Nucl. Inst. And Meth. In Phys. Res., A393, pp. 170-177 (1997).

3. Alex H. Lumpkin, "Beam Diagnostics for Future FELs," in Free-Electron Laser Challenges, SPIE, Vol. 2988, (Eds. P. G. O'Shea and H. E. Bennett), pp. $70-77$ (1997).

4. G. Neil, "Photon-and Electron-Beam Characterizations (WGVII)," Future Light Source Workshop, April 6-9, 1999, Argonne National Laboratory (2000).

5. Walter Barry, "Measurement of Subpicosecond Bunch Profiles Using Coherent Transition Radiation," Proc. of the 1996 Beam Instrumentation Workshop, May 6-9, 1996, Argonne, IL, AIP Conf. Proc. 390, pp. 173-185 (1997).

6. Yukio Shibata et al., Phys. Rev. E52, pp. 6787-6794 (1995).

7. A. H. Lumpkin et al., "Electron Beam Bunch Length Characterization Using Incoherent and Coherent Transition Radiation on the APS SASE FEL Project," Proc. of the 1999 International Free-Electron Laser Conference, Nucl. Inst. and Meth. in Phys. Res. (in press).

8. R. Lai and A. J. Sievers, "Determination of Bunch Asymmetry from Coherent Radiation in the Frequency Domain," AIP Conf. Proc. 367, pp. 312-326 (1996) and references therein.

9. J. Lewellen et al., "Operation of the APS rf Gun," Proc. of the 1998 Linac Conference, Chicago, IL, August 23-28, 1998, pp. 863-865 (1999).

10. N. S. Sereno, M. D. Borland, A. H. Lumpkin, "Setup of the APS rf Thermionic Gun Using Coherent Transition Radiation to Produce High Brightness Beams for SASE FEL Experiments," submitted to Linac 2000, August 14-21, 2000, Monterey, CA.

11. D. Rule, "Argcdr5.sm," August 1999.

12. N. S. Sereno, private communication. 